\title{
Cancer chemotherapy failure: a synthetic view
}

\author{
Falha em quimioterapia para câncer: uma visão sintética
}

Matheus Zanelatto Junqueira ${ }^{1}$, Roger Chammas ${ }^{2}$

Junqueira MZ, Chammas, R. Cancer chemotherapy failure: a synthetic view / Falha em quimioterapia para câncer: uma visão sintética. Rev Med (São Paulo). 2018 March-Apr.;97(2):141-53.

\begin{abstract}
Cancer is a highly prevalent and fatal disease, being one of the main causes of death in Brazil and in the world. In the last decades, a great advance has been reached in fight against cancer, with some translation in curability. However, these advances are still insufficient, and the prognosis of the cancer patient is usually unfavorable. Indeed, there are still many gaps in our knowledge about this complex and heterogeneous disease. Several treatments approaches against cancer are available to clinical practice - and some others are being developed - one of which is chemotherapy, both traditional and targeted therapy (TT), used - above all - as primary treatment for metastatic or secondary treatment in local or locally advanced disease. Several factors can act through many mechanisms to determine the failure of chemotherapy treatment. These factors are distributed across the various biological scales, from the molecular to the socioeconomic level, making the holistic view of treatment a great challenge and impairing the awareness of what can go wrong. The purpose of this paper is to be a comprehensive - but not superficial - picture of the many factors that influence the success of chemotherapy. In this way, the result of this work was this discussion on chemotherapy failure, were it was exposed recent advances, challenges to be overcome and new paths to be explored on this field.
\end{abstract}

Keywords: Neoplasms; Treatment failure; Drug therapy; Precision medicine.
RESUMO: O Câncer é uma doença de alta prevalência e letalidade, sendo uma das principais causas de morte no Brasil e no mundo. Nas últimas décadas, grandes avanços foram alcançados na luta contra o câncer, com alguma tradução em curabilidade. Contudo, esses avanços ainda não são suficientes, de modo que o prognóstico do paciente com câncer ainda é, geralmente, desfavorável. De fato, há ainda diversas lacunas em nosso conhecimento a respeito dessa complexa e heterogênea doença. Diversos tratamentos contra o câncer já estão disponíveis à prática clínica - e outros ainda estão sendo desenvolvidos - uma delas a quimioterapia, tanto a tradicional quando a alvo dirigida, utilizada, sobretudo, como tratamento primário contra doença metastática ou secundário contra doença local ou localmente avançada. Diversos fatores podem atuar, através de vários mecanismos, de modo a determinar a falha do tratamento quimioterápico. Esses fatores estão distribuídos ao longo de diversas escalas biológicas, do nível molecular ao socioeconômico, tornando uma visão holística do tratamento um grande desafio, prejudicando a compreensão acerca do que pode dar errado. O objetivo deste trabalho é ser uma leitura compreensiva - mas não superficial - dos muitos fatores que influenciam o sucesso de quimioterapia. Deste modo, o resultado foi a presente discussão, na qual foram expostos avanços recentes, desafios a serem superados e novos caminhos a serem explorados na área.

Descritores: Neoplasias; Falha de tratamento; Quimioterapia; Medicina de precisão.

1. Undergraduate Medicine student, Faculdade de Medicina da Universidade de São Paulo. São Paulo, Brazil. ORCID: 0000-00025478-5283. Email: matheus.zjunqueira@fm.usp.br.

2. Professor of Oncology, Department of Radiology e Oncology, Faculdade de Medicina da Universidade de São Paulo, Instituto do Câncer do Estado de São Paulo. São Paulo, Brazil. Advisor and chief of the Center for Translational Investigation in Oncology. ORCID: 0000-0003-0342-8726. Email: rchammas@usp.br.

Address: Matheus Z. Junqueira. Rua Conselheiro Pedro Luís, 300. Santana. São Paulo, SP, Brazil. CEP: 02020-050. 


\section{INTRODUCTION}

Cancer is a highly prevalent disease with high mortality rates. In the United States alone, data from the American Cancer Society estimates nearly 1.7 million new cases by $2017^{1}$, and in Brazil the National Cancer Institute José Alencar Gomes da Silva (INCA) has estimated almost 600,000 new cases by $2016^{2}$. The World Health Organization (WHO) has attributed 8.8 million deaths to cancer by $2015^{3}$. Mobilization for research, development and implementation of strategies for prevention, early diagnosis and more effective treatments - worldwide and at the national level - is impressive, and much has already been achieved. However, the prognosis of the cancer patient is still usually poor.

In fact, the word "cancer" includes a diverse set of biologically and clinically complex diseases with great heterogeneity and characteristics that promote intrinsic difficulties to the treatment - such as the fact that tumor cells originate from the patient's own healthy cells. As heterogeneous and complex as the disease is its treatment, counting on numerous weapons and combinations, often all of them insufficient. Several processes can undermine the treatment and lead to its failure.

Basically, there are local and systemic approaches of treatment. The local ones are surgery and radiotherapy, and still represent the best possibility of cure, but its effectiveness is restricted to localized disease ${ }^{4}$. Systemic approaches include chemotherapy - both traditional chemotherapy and targeted therapy (TT) - hormone therapy and immunotherapy.

Chemotherapy is the most well established strategy as primary treatment for metastatic disease, or as secondary treatment - in the adjuvant or neoadjuvant setting - for local or locally advanced diseases ${ }^{5-7}$. This paper will present a comprehensive overview of chemotherapy, pointing out determinants for its success or failure, for which health professionals and researchers should be aware. These factors discussed here operate through several mechanisms, at various biological levels, from the molecular to the socioeconomic level. The distribution of these factors along such distinct scales make the holistic view of treatment a great challenge especially in the highly specialized context of oncological research and clinical oncology - impairing the awareness of what can go wrong.

Our goal is, therefore, to produce a complete and succinct material capable of transmitting - in a single reading - the state in which research and clinical practice are at in regard of the fight against cancer through chemotherapy, in order to suggest research perspectives both basic and clinical - to help building evidence towards optimal clinical practice. Other treatment approaches (as surgery and radiotherapy), as well as the combination of two or more approaches (e.g. particularities of surgery followed by adjuvant chemotherapy), are not the scope of this paper and will not be discussed.

\section{METHODOLOGY}

This work is a bibliographical review of the scientific and academic literature regarding clinical and basic sciences aspects of chemotherapy and oncology, resulting in a critical synthesis of what is already known and applied - whether in research or in medical practice with focus on mechanisms throughout treatment failures may happen in chemotherapy. However, it is important to emphasize that this work is not a systematic review. Therefore, there were no specific search parameters, nor inclusion and exclusion criteria. Sources were reached through PubMed and renowned oncology textbooks.

\section{General mechanisms and factors implicated in treatment failure}

Chemotherapy - both traditional cytotoxic and TT - may have its success rates hampered by many factors ${ }^{5-7}$. These include biological, clinical, socioeconomic and psychological aspects. Interactions between these factors are key in determining treatment failure, and highlight the need for new therapeutical options (pharmacological and otherwise).

For a patient with a given type of cancer to receive successful treatment, his or her diagnosis must firstly be correctly made. This implies the existence and availability of the appropriate laboratory, imaging and anatomo-pathological studies, with good specificity and sensitivity, which must be sufficient for determining histological grade and staging of the patient's neoplasm. The great diversity of types of cancer and, for each type, the molecular heterogeneity of its subpopulations, are two factors that implicate the need for more and more precise diagnostic procedures ${ }^{9}$. This results in a great amount of scientific and financial effort implicated in their wide application to the general population ${ }^{9}$.

Ideally, the most effective treatment available should be administered as soon as the diagnosis is determined, because an early treatment is key to increase therapeutical success and to prevent relapse $\mathrm{e}^{5}$. In the case of cancers for which there are well-established and evidencebased screening procedures, such as those currently used for colorectal and uterine cervix cancers, early treatment is dependent on the availability and correct application and evaluation of the results of those screening procedures. The development of such procedures for other cancers, at a nation-wide level, is hampered by the need to identify reliable biomarkers appropriate for diagnostic usage for each type of cancer. However, using these biomarkers as standard diagnostic procedures in the general population is also challenging due to their usually high costs ${ }^{9}$. 
After the correct diagnosis has been made, an existing treatment must be made available by the patient's healthcare provider, both physically and financially, for as long as it takes to achieve therapeutical success. This may imply frequent travels, moving and the need for a family member or friend (part of the patient's social support network) to be with the patient along the course of the treatment. It is crucial that the patient is made aware, after his or her diagnosis has been determined, of the available therapeutical options, as well as of his or her prognosis as far as those are predictable ${ }^{9}$. Most importantly, the patient, aided by his or her social support network, must be psychologically willing to undergo the chosen treatment ${ }^{10}$.

Due to the genomic instability of cancer caused by the failure of DNA repair mechanisms (e.g. BRCA1 and 2 pathways in breast cancer), selection of neoplastic cells resistant to treatment is frequent, which leads to the problem of tumor resistance and calls for the development of new therapies. The tumor microenvironment also plays a role in the genesis of resistance, since it may impair both the local absorption of anti-cancer ${ }^{11}$ and immune cell functions in anti-tumor response $\mathrm{e}^{12,13}$ (e.g. up-regulation of CTLA4 in some forms of melanoma).

Follow-up during and after treatment is a major concern, which brings back the issue of possible travels and the need for the patient to move. Long-term treatment - such as hormone therapy after receptor-positive breast cancer excision - must also be available and accessible to the patient at his or her place of residence. The patient's social support network plays an important psychological and sometimes financial role in enabling treatment success.

Complications and other consequences of the disease, both somatic and psychological, must also be identified and correctly dealt with - besides, whenever possible, prevented - by the healthcare professionals, as well as possible worsening of pre-existing clinical conditions due to cancer or to cancer treatment. Multimorbidity, especially in older patients, significantly worsens outcome for cancer patients, due to the reduced therapeutical options often available ${ }^{8}$.

\section{Chemotherapy}

Chemotherapy is didactically divided into two domains: traditional and TTs, mostly due to the latter's particular development process ${ }^{5-7}$.

Traditional chemotherapy drugs, which were first developed in the second half of the XX century, were originally discovered through the evaluation of the toxic effect of certain substances on tumor tissues relative to normal tissues. Some of the first chemotherapy drugs were derived from mustard gas, in studies led by groups that originally worked with antimicrobial drugs, thus being aimed at killing pathogenic cells (in the case of infectious diseases, external biological agents) or at interrupting their proliferation to treat disease $\mathrm{e}^{5,7}$.

TT drugs, on the other hand, are developed with a target in sight - in other words, they are designed to act upon a specific component of a molecular pathway or process which is known to be altered in cancer. This process is typical of the post-genomic and proteomic revolution era, whose great technical advances led to significant accumulation of knowledge on metabolic and signaling pathways altered in cancer ${ }^{9}$. Molecularly targeted drugs can be split into two categories: monoclonal antibodies (mAbs) and tyrosine kinase inhibitors (TKIs) ${ }^{14}$.

When it comes to chemotherapy, it is important to point out that, with rare exceptions (such as choriocarcinoma $)^{5}$, isolated currently used drugs, in secure doses, are unable to cure cancer. Thus, in many cases a combined approach is superior to an isolated drug since it enables maximum cytotoxic effect in the therapeutical window $^{5-7}$. This is result due to the simple summation of the effects of the drugs involved, synergism - one drug enhancing the effect of another - and due to being more molecularly comprehensive in terms of the heterogeneity of the tumor cell population profile, avoiding and/or slowing tumor resistance emergence.

Some general principles must be observed in combined chemotherapy ${ }^{5,7}$, and if not respected, treatment failure may occur due to direct and/or indirect mechanisms. They are: (i) only drugs which are effective against the patient's kind of tumor must be part of the combination; (ii) there should be no toxicity overlap - when choosing between two drugs of the same profile, the one with best isolated efficacy should be chosen - which leads to a greater variety of adverse effects but prevents lethal consequences; (iii) the drugs should be administered in their ideal maximum dosage; (iv) biochemical interactions among combination drugs must be known; no dose is to be arbitrarily reduced, nor should a drug be omitted.

Another important concept in chemotherapy, reached through models of tumor kinetics and tumor drug response - further explained forward - is log kill. Log kill predicts that a specific agent at a specific dosage will kill a regular logarithmic number of cells, despite the absolute number of tumor cells. Thereby, with the log kill of an agent at some dosage is 3 , it will eliminate $10^{3}$ cells of the tumor burden, either $10^{9}$ to $10^{6}$ or $10^{5}$ to $10^{2}$ cells ${ }^{7}$. The resulting effect is the inverse relationship between tumor burden and curability ${ }^{7}$. As consequence, one of the reasons of chemotherapy failure is that the treatment begun when the chemotherapeutic agents available were no longer able to eliminate all tumor cells. Other concepts are associated with this last statement, which will be presented later along with tumor kinetics.

Currently chemotherapy can be used, in the treatment setting as a whole, in four configurations: primary treatment, neoadjuvant treatment, adjuvant treatment and palliative treatment ${ }^{7-9}$. 


\section{Toxicity to normal tissues}

Chemotherapy drugs in cancer treatment act by killing and/or stopping the proliferation of tumor cells, leading to the reduction and, if possible, to the complete elimination of the tumor mass. In order to achieve that goal, chemotherapy uses substances with significant cytotoxic properties.

In contrast with antimicrobial chemotherapy, in antineoplastic chemotherapy biological similarities between the target (tumor) and the normal cells are significantly greater. Thus, the toxicity of these drugs also hits normal cells; regardless of the drug being traditional or TT, it has a toxicity profile - linked to its pharmacological mechanisms - both for normal and tumor tissues.

This toxicity profile may be represented by a doseresponse sigmoid curve in which the toxicity (expressed through cell death) increases according to dose, following the phases of latency, linearity and stabilization, also having an action threshold ${ }^{5,7}$. The difference among these curves is the therapeutic window of the drug (Fig 1). For common chemotherapy drugs, the therapeutic window of the drug is often very narrow, which leads to a very thin line between what is effective and what is detrimental in terms of treatment. The therapeutic window is not the same for all patients; one must bear in mind that each individual has a different status of their organic systems, regarding functional reserves, preexisting diseases and other limitations. Thus, an overly harmful dosage for a patient may be, and will be, different from that of others. If the functional reserve of the patient is reduced, due to age or to disease, his or her therapeutic window is narrower than is that of other patients. In the case, for instance, of a cancer patient with a history of diabetes mellitus and a diabetes-secondary nephropathy, he or she will withstand limited doses of drugs with a nephrotoxic profile if compared to other non-diabetic patients.

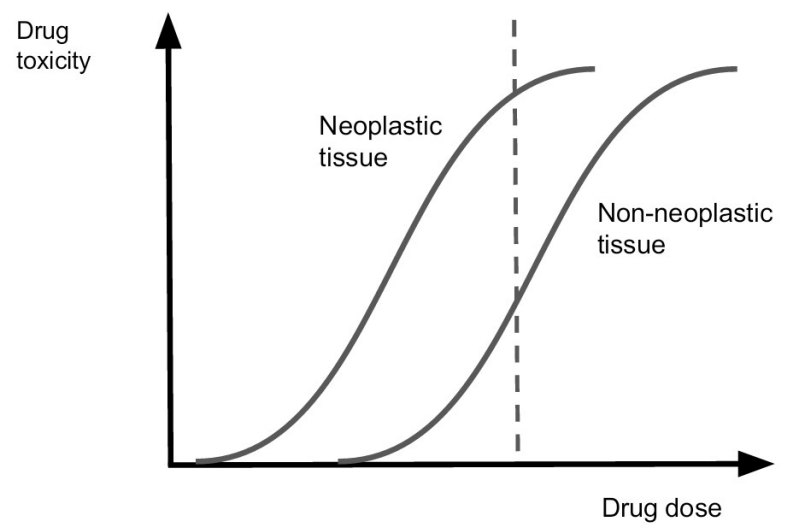

Figure 1. Graphic representation of the sigmoid dose-response curves of neoplasic and non-neoplasic tissues. To a given dosage, the vertical distance between the two curves represent the therapeutic window

\section{Adverse effects}

As a consequence of drug-induced cell death and of other processes due to the pharmacology of each drug, chemotherapy implies a clinical toxicity - that is, adverse reactions. These play an important role in altering the patient's quality of life (QL), and may themselves be target of a clinical intervention.

There are many ways to measure these adverse reactions in order to define a drug's toxicity profile. The most commonly used is the National Cancer Institute's Common Terminology Criteria for Adverse Events $(\mathrm{CTCAE})^{15}$. Among these Criteria is a recommendation for the term "toxicity" not to be used, due to the lack of a precise definition, despite the historic use of this term the CTCAE itself was named Common Toxicity Criteria until the $2^{\text {nd }}$ version, after which it became CTCAE.

Adverse effects also involve risk factors, including genetic ones. Some of them are easy to infer from the clinical setting, such as previously diagnosed liver damage in the case of a drug that has considerable hepatic metabolism. Genetic risk factors such as drug metabolism enzyme polymorphisms, on the other hand, are difficult to infer from clinical data, and thus must be actively searched ${ }^{16}$. The search for risk factor-associated polymorphisms would be coherent with the concept of personalized medicine, but is, in the context of current clinical practice, hard to put in place.

The importance of adverse reactions in chemotherapy has shaped many of the principles that govern it - suffice it to analyze the space dedicated to their management in the oncology literature. There are three main consequences of chemotherapy related to adverse reactions: (i) impossibility of treatment, (ii) undue adjustments in dosage and treatment regimen and a (iii) negative impact in the patient's QL and mental health. All of these factors can contribute to treatment failure.

\section{I - Impossibility of treatment}

A drug's toxicity can result in clinically significant adverse effects in such a way that the patient is physically unable to withstand them. In that case, the use of that specific drug is impossible, as well as in the case of preexisting clinical conditions that would be aggravated by the drug. The use of a specific drug may also become impossible during treatment as a result of the deterioration of the patient's health status, both due to the use of the drug and to other reasons.

Usually, when a therapeutical option (drugs, combinations and treatment regimen, concepts which will be described below) is discarded due to the aforementioned reasons, the alternative treatments bring worse results. In extreme cases, the alternative is nonexistent or inefficient, with greater impacts on the patient's QL and less clinical benefit. Besides, reducing the available drugs also reduces 
the possibilities of combinations, which impairs the efficacy of treatment and increases the risk of developing resistance, further described below.

It is also important to highlight that palliative care is a treatment approach. It is not an approach that pursuit's cure; instead, it searches for relief of the suffering associated with terminal conditions ${ }^{8}$. In this way, incurability does not implicate impossibility of treatment, as long as the palliative chemotherapy is an elective option. Although palliative care and chemotherapy may seem divergent concepts, considering the amount of adverse events and loss of QL commonly associated with this type of treatment, there are some practical examples of this approach. For instance, palliative chemotherapy of pancreatic cancer is used to increase QL and overall surviving time, despite any speciation of cure ${ }^{4}$.

\section{II - Undue adjustments in dosage and treatment regimen}

The therapeutical dosage of most chemotherapy drugs falls within the linear phase of the dose-response curve $^{5}$, which has significant linear variations in response with slight changes in dosage 7 . Thus, small decreases in dosage may lead to a significant loss of therapeutical efficacy, which has been finally demonstrated in 1994 by Wood et al. ${ }^{17}$.

Empirical dose reduction may easily be incorporated in a professional's clinical practice as a way to reduce side effects in order to keep the patient from a loss in his or her QL. Such reductions are an important mechanism of treatment failure ${ }^{5,7,17}$. A reduction in the dosage of a nephrotoxic drug made, for instance, aiming to preserve a patient's renal function, may determine treatment failure.

Treatment regimens: Regarding the treatment regimen, an important concept is that of dose intensity, as proposed by Hryniuk et al. ${ }^{18,19}$, which can be defined by the amount of the drug during the course of time. The standard type of treatment, determined by the direct relation between dose and tumor cell death, is called doseintense chemotherapy, using the maximum tolerated dose (MTD). Besides the simple drug concentration, MTD takes into account other pharmacokinetic issues which will be further discussed below.

Two treatment regimens involve MTD. The first one is the alternate regimen, which was proposed based on an experimental model proposed by Goldie and Coldman et al. ${ }^{20}$ to explain the development of resistance. The second one is the sequential regimen, proposed by Norton and Day et al. in $1991^{21}$ based on a revision of the Goldie and Coldman's tumor kinetics model, proposed by Norton et al. in $1988^{22}$. The alternate regimen consists in alternating the cycles of drugs without cross-resistance, whilst the sequential regimen the cycles of a single drug should be ended before those of another can start (Figure
2). To this day, no consensus has been reached on which regimen is the most efficient ${ }^{23-25}$, and finding a single formula to face all types of cancer is unlikely ${ }^{26}$.

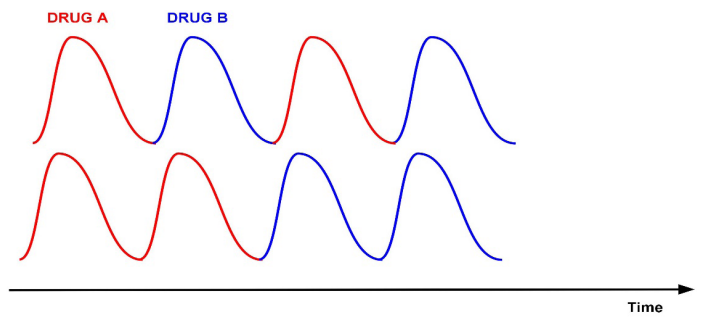

Figure 2 Graphic representation of the alternated regimen (upper curve) and sequential regimen (down curve), both MTD approaches

Currently, using concepts from studies on the tumor microenvironment, a third regimen is being proposed: the metronomic regimen (Figure 3). It consists of lower doses, so as to enable a higher frequency and length of application, in regular cycles destined to promote less toxicity, induce less resistance and improving anti-tumor immune response ${ }^{27,28}$. However, it remains an experimental concept with some intrinsic limitations, such as in the case of disease with needs of rapid regression (e.g. some cases of glioblastoma).

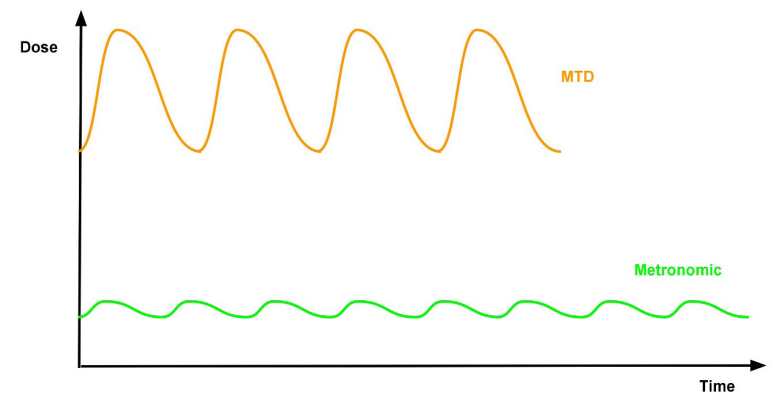

Figure 3 Comparison between MTD (in orange) and metronomic regimens (in green)

Given cancer's heterogeneous characteristics, it is highly unlikely for a single treatment regimen to be the most efficient for all types of malignancies ${ }^{28}$. For instance, using MTD against leukemia in children is highly effective: however, the same regimen shows lower success rates on more intrinsically heterogeneous malignancies, such as small cell lung cancer. Studies led by Goldie and Coldman in the $1980 \mathrm{~s}^{26,29}$ had pointed out that aspect and drew attention to the need to ascertain the efficacy of the alternate regimen in each type of cancer. 


\section{Perspectives: complex modeling and personalized medicine}

There is a hierarchy between the importance and fidelity of different measures of the concentration of a drug. Active intracellular drug concentration bears a closer relation to the therapeutical response than does the plasmatic concentration or even dosage ${ }^{16}$. In the clinical context, however, the direct measurement of the most relevant compartments is often impossible.

In order to overcome that shortcoming, pharmacokinetic models are used during drug development as indirect measurements of these local concentrations. Population pharmacokinetic models were introduced by Lewis Sheiner in $1972^{30}$ and evolved, along with information technology, leading to current models including pharmacodynamics, tumor kinetics, cell biology and tumor microenvironment data, which enable those models to make outcome and adverse effect-related predictions.

Pharmacokinetic and tumor kinetic modeling are complex concepts and are not currently part of the clinical practice in Brazil, and is more widespread in the academic context. Nevertheless, some models destined for clinical use have been presented, such as $\mathrm{EMMA}^{31}$ (ex vivo Multiple Myeloma Adviser), which is able to provide, within five days, a prediction of success for different drug combinations and isolated drugs.

One way these models can be of use in the immediate oncology context is through guiding researchers through the countless data they compute, working as screening tests of drug combinations and treatment regimens with greater potential, so that they may be validated and applied in the clinical practice.

The many variables included in pharmacokinetics and treatment regimen lead to countless approaches, which can be previously analyzed with computational models to ascertain how promising they might be.

\section{III - Interactions between social, psychological and biological factors and their impacts \\ In the current medical context, the need for} taking psychosocial aspects into account in health care and attention to mental health have become undeniable. Cancer has high mortality and morbidity rates, with severe impacts in patients' QL, and often lead them to face death ${ }^{32}$. Both these aspects contribute to form a social stigma around the disease ${ }^{33}$, which has already been called "Emperor of All Maladies" 34 and that worsens even further its negative impacts on patients' mental health, and even on their families and friends ${ }^{10}$.

Both mortality and morbidity may be due to the disease itself or to the treatment, due to its toxicity in the case of chemotherapy. Adverse effects contribute to high morbidity rates in cancer patients, which impacts directly on QL and mental health, leading to the reduction of mobility, social isolation, stress, anxiety, depression and sleep and eating disorders ${ }^{32}$. All of those conditions communicate in a vicious way, making a complex network. Therefore, the link between cancer and mental health and the impact of mental health in the process of the disease, as well as bio-behavioral mechanisms attributed to that impact, shall be discussed further below.

Despite being a serious disease, depending on intervention and often having poor prognosis, cancer has a mental health aspect that cannot be neglected, since that aspect is part of the patient's health as a whole.

Studies show that $35 \%$ to $50 \%$ of cancer patients have psychic disorders, depression and anxiety being the most common diagnoses ${ }^{32}$. Such disorders spring from many factors, such as fatigue, pain (from the disease and from its treatment), disabilities, functional losses and incapacity, culminating in impairments in basic and instrumental daily activities, stress due to worrying about the disease, fear of dissemination of the disease, of relapse and of death, feelings of guilt, powerlessness, sadness, confusion, stress due to financial questions, unemployment, income reduction and high medical costs, inability to make future plans and other situations ${ }^{10}$. The mechanistic discussion of the aforementioned factors escape the scope of the present study, but their complexity - and quite often their simultaneity - highlight the importance of taking them into account and of their approach by the healthcare professionals. It is important to point out that many of these conditions may be present in the life and influence the health of the person before the illness, and become more significant after the cancer diagnostic. health

Impacts of disease and treatment on mental

Even though playing a significant detrimental role in patients' health (including physical) and QL as a whole, psychological disorders are underdiagnosed in cancer patients ${ }^{10,35}$. The reasons for that situation are multiple and often overlapping.

Studies show that less than a quarter of all patients have explicit psychological complaints, and half of them claim that these subjects are irrelevant to doctors, and almost $20 \%$ feel discouraged to voice these complaints ${ }^{32}$. Besides, doctors seldom actively search psychological disorders, and most of all when they feel unprepared to deal with the dense emotional burden of the disease ${ }^{32}$. These data reflect a culture that values somatic health in detriment of mental health, while increasingly stronger evidence show an intricate relation between the two aspects, as will be explained further below. In its 1946 Constitution, the World Health Organization highlights that relation in its definition of health - a state of complete physical, mental and social well-being".

Curiously, in a study on the needs reported by 
patients ${ }^{36}$, seven out of the ten most frequent needs belonged to the psychological field, and the remaining three were linked to the desire for more information on disease and treatment.

It is important to state that the wish for information may have a positive impact on the patient's mental health, if fulfilled, and a negative one if ignored, above all if it is related to a feeling of powerlessness and to satisfaction with the treatment ${ }^{37}$. In that sense, providing information to the patient, while respecting their demands and their readiness to receive it, and their participation in decisionmaking related to their treatment has a positive impact in their mental health ${ }^{37}$. Even when the patient report that he didn't wishes to have control of his treatment, patients reported satisfaction with care is improved when the physician applies shared decision-making techniques ${ }^{37}$.

The role of communication, both in the investigation of psychiatric disorders from indirect signs and complaints and in the better transmission of information and perception of the demand for information, is fundamental. It also demands significant preparation by the healthcare professional. Nonverbal aspects of everyday communication may have a great impact in that context, such as whether the doctor is standing or sitting when delivering information, and also the relation between the doctor and the computer in that communication situation with the patient ${ }^{38}$.

Thus, the current concepts of patient-centered medicine and evidence-based medicine are headed toward the same track: the need for better preparation of doctors and other healthcare professionals for dealing with mental health, communication and humanized healthcare ${ }^{36,38}$.

\section{treatment \\ Impacts of mental health on disease and}

As mentioned above, there is growing evidence of the inseparability between somatic and mental health ${ }^{10,32,33,35-43}$. Currently there are two well-established mechanisms that bridge the psychological and the biological: activation of the sympathetic nervous system (SNS) and of the hypothalamus-pineal-adrenal axis (HPA), mainly through the elevation of cortisol levels ${ }^{39,40,41}$.

Those two mechanisms, along with psychological factors, may lead to habits and behaviors that are harmful to patients' health, such as obesity, sleep disorders, alcohol and tobacco use and other situations ${ }^{35,39,40}$. Three other aspects, more strictly biological, are the reduction of immune response, the high levels of DNA damage with less repair activity and the increase in levels of VEGF (vascular endothelial growth factor) ${ }^{40}$.

Immune response reduction seems to be associated both with behavioral aspects (worse quality of sleep, less aptitude for exercise, alcohol and tobacco use and inadequate diet as ways of compensating for increased stress) and with the two aforementioned neuroendocrine axes. The SNS elevates seric levels of catecholamines and the HPA axis does the same for cortisol and other glucocorticoids, both physiological signals of stress ${ }^{39,40}$. These neuroendocrine factors induce an inhibition of apoptosis and a suppression of immune response through the reduction of the proliferative capacity of lymphocytes and other markers, such as NKCC (natural killer cell cytotoxicity $)^{40}$, which correlates to metastatic patients' survival and metastization chances ${ }^{44,45}$.

In a more direct way, these axes reduce the DNA repair cellular activity, worsening the genetic instability associated with cancer pathogenesis and tumor progression and increasing malignity and the development of resistance. Besides, greater DNA damage and telomerase activity, leading to the promotion of an “immortal" phenotype, are also implicated ${ }^{39,40}$.

Finally, studies show that stress-induced activation of the SNS, leading to an increase in $\beta$-adrenergic stimulation, leads to an increase in VEGF levels, which culminates in greater tumor vascularization, enabling the tumor to grow more, and greater tumor mass ${ }^{41}$.

Therefore, since mental health plays a role in immune suppression and in the increase and progression of tumor vascularization, it becomes an important spot for treatment failure. Animal ${ }^{42}$ and human studies aimed at direct (e.g. depression and mortality ${ }^{35}$ ) and indirect (e.g. stress and $\mathrm{NKCC}^{39}$ ) relations highlight that importance. Besides, there is evidence supporting a role, through these same mechanisms, of mental health in cancer pathogenesis $^{44}$.

Fortunately, mental health is manageable with current technological resources, if compared, for instance, to mechanisms related to tumor resistance, which aren't yet thoroughly clarified and whose interventions are still being developed. This affirmation is based on the fact that psychiatric interventions in cancer patients have not only been proven to improve depression and anxiety but have also been linked to increases in immune response biomarkers and to decreases in cortisol and catecholamines $^{33,39,46}$.

\section{Drug insensitivity}

In TT, an altered molecular pathway, usually a signaling one, is the target ${ }^{47}$. Therefore, for the treatment to be efficient, that pathway must be altered in the patient. Since cancer is a heterogeneous disease, not all patients display the target alteration. Besides, even in patients that have the alteration, it will not be present in all cells or elements, such as the extracellular matrix and nontumor associated stromal cells, such as tumor associated macrophages and endothelial cells.

Additionally, given that there is a need for an altered molecular pathway for the treatment to work, there is a need to verify the status of that pathway in the patient. That is done through the use of biomarkers which must 
be able to identify if a patient will respond to a specific treatment.

\section{Biomarkers paradox}

Biomarkers are measurable factors ${ }^{9,47,48}$ that can report information on a given physiological or pathological process. Ultimately, all biochemical tests used in the clinical context have the same principle as that of biomarkers. However, in this study, we focus on modern (post genomic and proteomic revolution) biomarkers, which are among the great promises of personalized medicine.

Being laboratory tests, biomarkers, are subject to false positives and false negatives. Usually, tests that are too sensitive lack specificity, and vice-versa, and depending on these two aspects, a biomarker-associated test may be more indicated for some uses than for others. As a general rule, highly sensitive tests are used for screening, whereas highly specific tests are used to determine a diagnostic ${ }^{49}$. Using a biomarker detection test that is too sensitive poses the risk of indicating treatment to patients which will, at best, not benefit from it, while a highly specific biomarker test could fail to indicate treatment to patients that would respond to it.

Another important issue related to biomarkers is that they display some specificities in their development which make it especially slow and difficult - and also, in some cases, poorly attractive to the pharmaceutical industry. This has been referred to as the biomarkers paradox $^{9,48}$.

\section{Insensitivity}

With the advent of TT, drugs were developed aiming at tumor biology features that were different from normal tissue. Therefore, theoretically, the drugs obtained would have greater therapeutic windows. On the other hand, the development of targeted drugs implicates that most patients are intrinsically insensitive to those drugs, since these are specifically designed to act on signaling pathways which are altered only in patients with a specific kind of cancer, and not in most patients. This has implications on the validation of that type of therapy for general population use, since the high cost of development and application of drugs that are directly beneficial only to a limited number of patients must be confronted with other priorities concerning the health of the general population ${ }^{50,51}$.

Insensitivity is one of the major biological mechanisms for TT failure, along with resistance. Another mechanism is the high cost of those therapies, both for the drug itself and for the implications that the use of biomarkers implies. The burden of the high cost is worsened if one takes into account the populational restrictions that insensitivity imposes - high cost, few patients receive benefit). That discussion is linked to personalized medicine and involves not only medical practice but also public health, administration and the pharmaceutical industry.

TT drugs have more specific mechanisms of action, they bear a closer relation to insensitivity. Regarding traditional chemotherapy drugs - in which the action mechanisms are broader - therapeutical inefficiency is more related to resistance than to insensitivity, and thus analog mechanisms for those drugs will be addressed along with other aspects of resistance. Although, there is an important exception: many traditional chemotherapeutic agents acts to DNA damage and disrupting DNA replication. In this way, their therapeutical effect holds tight relationship with the cell cycle. Thereby, is reasonable to state that a population of tumor cells that are not replicating are insensitive to a traditional agent (as long as its mechanism of action is influenced by the cell cycle). As a consequence, traditional cytostatic drugs, or TT drugs that aim and inhibit the replication signaling and mechanism, may have an opposite effect in comparison to traditional cytotoxic drugs and other TT drugs, and this to grups may not be associated in a same chemotherapy regime.

Together with those intrinsic mechanisms, a more extrinsic form of insensitivity is the constitutional impossibility of the drug to reach its target, such as, for instance, a drug aimed at controlling a tumor in the central nervous system but unable to cross the blood-brain barrier. Independent of the mechanism, the effect of insensitivity leading to therapy failure is the same.

\section{Resistance to drugs}

Tumor cell kinetics and emergence of resistance

Tumor growth rate and pattern have great clinical relevance since many chemotherapy drugs disrupt proliferation, and thus must be administered in periods with high proliferative activity. This is particularly relevant in the traditional chemotherapy, since many of the traditional agents acts - in some instance - through DNA damage or DNA replication impairment (e.g. alkylating agents, platin derivatives, nucleoside analogs, anthracyclines and topoisomerase inhibitors).

The quantitative study of tumor growth began in the 1950s by Collins et al, who proposed a constant duplication time for cancer, which led to an exponential model for proliferation ${ }^{52,53}$. Their theory was heavily criticized in the following decades, above all regarding the fact that not all cancers would behave the same way. Steel et al. pointed out in 1977 that in some primary fibroadenoma samples in rodent models had irregular growth patterns.

Retsky et al. ${ }^{52}$, in 1990, compared the applicability of two tumor kinetics models: Gompertzian, a modification of Collins' exponential model that takes into account the 
tumor's proliferative ability and nutrient availability in the space available for proliferation, and an irregular model, which combined some gompertzian-like growth interspaced with periods of no growth. This second model was initially described a few years before in breast cancer models but was later extended for intestinal, rectal and lung cancers.

Later, other models were described, such as power law and von Bertalanffy's, and for different cancers, different models gained more acceptance. Benzekry et al. $^{53}$, in 2014 , evaluated many parameters of these models, mainly their descriptive value as compared to lung cancer, and came to the conclusion that for lung cancer, Gompertzian and power law were among those with the best descriptive value, whereas for breast cancer, exponential-linear models showed better descriptive power.

To expose some concepts, we will adopt the Gompertzian model (Figure 4). In this model, the growth fraction rate decreases with the tumor burden, having its peak at the first third of the curve, way before clinical symptoms and detection ${ }^{7}$. As a result, when most of the treatments occur, the tumors are already with decreased growth rate and, thereby, with decreased sensitivity to cell cycle dependent drugs. Adding this to the concept of $\log$ kill $^{9}$ - in part, explained by this fact - the inverse relationship between tumor size and curability is reinforced. Along with metastatic dissemination - with consequent loss of curability by local therapies - and resistance, this is one of the most important mechanisms of treatment failure in advanced disease.

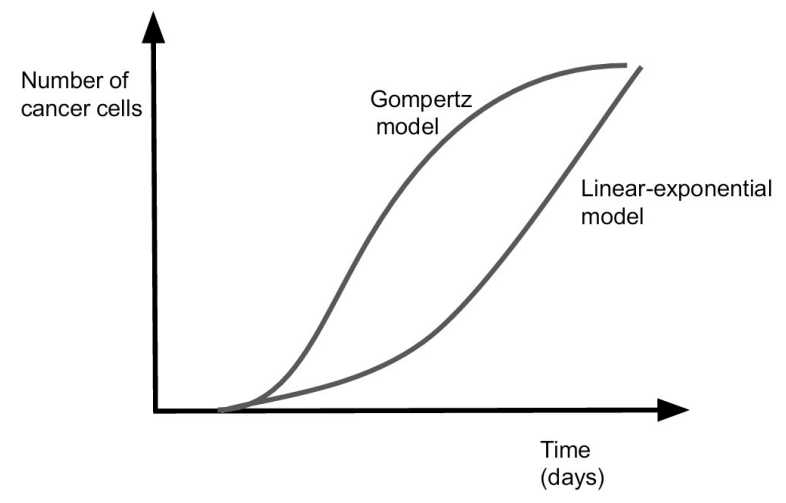

Figure 4. Graphic representation of tumor growth according a gompertzian like model and a simple linear-exponential model

Resistance is one of the most important mechanisms of treatment failure, and it may be primary or acquired. Primary resistance refers to lack of effectiveness of a given drug as result of a process that took place in the early tumorigenesis. As previously discussed, this situation is more closely related with insensitivity that with the common use of the resistance concept. The acquired resistance, on the other hand, results from a process that happened at some point of the tumor progression in association the emergence of a resistant cell line due to external selective pressure of a previous treatment. The ordinary use of the term "resistance" often refers to acquired resistance, and in this discussion they will be used as synonyms.

The general mechanisms leading to resistance arise at both oncological and non-oncological contexts, and include (i) diminished concentration of active drug on the target compartment (intracellular compartment, with exceptions); (ii) metabolic changes (activation of alternative pathways); and (iii) structural alterations of targets. Especially in the oncological context, a fourth broad mechanism is (iv) increased activity of survival and repair pathways and inhibition of cell death, especially apoptosis $^{54}$.

Metabolic changes refers to bypasses at production pathways of essential metabolites to tumor cell function. Together with structural alterations of targets, they represent mechanisms that are agent specific and private. As a result, they play important role in the resistance of TT and antimetabolites individually. On the other hand, diminished concentration of active drug and increased survival, repair and apoptosis resistance develops through mechanisms that are much less agent specific, contributing to resistance processes of several drugs at the same time and, possibly, installation of a multidrug resistance (MDR) phenotype.

The decrease of active drug concentrations may result of increased drug efflux and/or inactivation, decreased drug input (less relevant to MDR) and/or activation. The most important mechanism of increased drug efflux is the increased expression and activity of ATP-binding cassette transporters (ABC transporters), a large family of membrane proteins with great influence on the MDR phenotype, with highlight to $\mathrm{P}$ glycoprotein (P-gp). ABC transporters act a role in resistance to several different drugs ${ }^{11}$.

In increased drug inactivation and decreased drug activation, specifics enzymes plays important roles at the resistance mechanism to some drugs. However, a single factor influences several drugs when it come to drug inactivation: tumor microenvironment $\mathrm{pH}^{12,13,55}$. The characteristic acidification of the tumor microenvironment account for reduction of effectiveness of many drugs, throughout several mechanisms (e.g. increasing expression of $\mathrm{ABC}$ transporters or promoting increased genomic instability ${ }^{12,13}$ ), and also plays important role in immunosuppression ${ }^{13}$ - and the immune response after treatment have great importance to determine treatment success. The example of hypoxia acting as a single agent affecting action mechanisms of several drugs highlights the importance and complexity of tumor microenvironment, 
and the still poorly understood influence it probably has. Another very important mechanism related to MDR is increased processes of repair and survival and decreased processes of cell death, mainly apoptosis ${ }^{11}$. This is possible by the fact chemotherapeutic agents - traditional or target-oriented - act in such a way that the ultimate mechanism resulting in cytotoxicity is the induction of apoptosis. If apoptosis is inhibited, either by stimulation of survival, or by inhibition of pro-apoptotic factors, cytotoxicity is reduced. The enhancement of repair processes acts on the resistance of drugs whose mechanism of action involves DNA damage (e.g., platinum derivatives and alkylating agents). Alteration of these processes usually occurs in the signaling, for example changes in $\mathrm{Bcl} 2$, p53 and in the metabolism of ceramides ${ }^{56}$. These changes not only lead to resistance, but are also hallmarks of cancer, directly related to its pathogenesis and progression. Thus, they are intensively researched as targets for therapeutic intervention.

Through those mechanisms - as MDR phenotype or single drug resistance - resistance have great power to determine treatment failure. With the primary line of chemotherapy fail, the patient will need to recur to the second line of drugs, generally less effective, increasing the chance of the treatment -as a whole - to also fail. Other scenario is recurrence after remission, in which resistance manifests in great part of the recurrent tumors, since the cells that survive the first treatment and cause the recurrence are probably resistant. It is also important to point out that resistance does not necessarily implicate completely effectiveness loss of some drug. It may just narrow it down it therapeutical window, and together with the effects of adverse events, make the treatment impossible.

\section{Target-oriented therapy (TT)}

TTs display some particular features about its associated treatment failure mechanisms, which are insensitivity, discussed above, high cost and a particular susceptibility for resistance.

Out of these failure mechanisms, the most relevant one still is its high cost, since it impairs more significantly the clinical conduct for individual patients and restricts the target population of a high cost therapeutical option, which makes it less likely for this option to enter the Brazilian public health context. If such therapies are not available to patients, treatment failure becomes more likely due to the shortening of the available "therapeutical arsenal".

That is particularly relevant in cases of metastatic disease, in which treatment options are already inevitably systemic and usually have a poor prognosis. Disparities of access to trastuzumab - an anti-HER2 (Human Epidermal Growth Factor 2) monoclonal antibody used in HER2 positive breast cancer - is an important example of that treatment failure mechanism. Before that drug became available to the general population, the oncogenic protein it targets was known only to be an indicator of poor prognosis, resulting that chemotherapy in patients with HER2 positive breast cancer, without access to trastuzumab would probably fail. On the other hand, HER2 positive breast cancer patients with access to trastuzumab have increased treatment success rates.

Despite the aforementioned difficulties, it would be incorrect to say the use of TT is inexistent in the Brazilian public health system, SUS (Sistema Único de Saúde), tamoxifen, an estrogen receptor antagonistic prodrug, and trastuzumab itself being two examples of that presence. Besides, it would be incorrect to state that such difficulties affect only SUS or even Brazil ${ }^{51}$.

Similarly to the biomarker paradox, a TT paradox has been spoken of: despite being often mentioned as "the future of medicine", $90 \%$ of TT drugs undergoing clinical trials fail to reach approval for clinical use ${ }^{47}$. Among the contributing factors for that situation are: (i) high cost and long time for development and approval - both a cause and a consequence of said paradox - (ii) higher than expected toxicity, (iii) increased susceptibility to resistance, (iv) lack of suitable combination strategies, (v) insensitivity in many patients and, consequently, difficulty in the determination of treatment efficacy and in the determination of whether the patient belongs to the target population ${ }^{47}$. The increased susceptibility of TT to resistance arises from the high specificity of its mechanisms of action, and from the great heterogeneity of cancer. Thus, in the same way that the altered pathway targeted by a drug may not be so in a given patient, that same mutation may be present only in a subpopulation of the tumor - as indeed is the case in most situations, according to the current clonal succession and tumor progression theories use to explain tumor cell population dynimics $^{57}$.

After the beginning of treatment or after a first treatment is done, target cells may have been completely eliminated. However, the tumor can maintain a cell population above the minimal tumor cell load and return to its progression - but this time, with an acquired resistance to the drug in question due to selection of tumor subpopulations. That is an intuitive mechanism additional to other traditional chemotherapy resistance mechanisms. Adding itself to other mechanisms of resistance, it makes TT more prone to that process.

TT drugs' toxicity also calls for special attention. Theoretically, TT should limit toxicity and widen the therapeutic window; however, that has not been verified clinically - at least not with the expected intensity ${ }^{14}$. Two mechanisms are responsible for that: pleiotropic target or pathway (paradoxical toxicity) and off-target.

Pleiotropism refers to a molecule (or a molecular 
pathway) participating in more than one tissue, organ or pathway or function. Inhibiting the target molecule/ pathway, the drug may inhibit another physiological process with the same elements, or even the same physiological process, but in a different context where it is necessary rather than detrimental. This leads to the socalled paradoxical toxicity ${ }^{14}$. One example of that toxicity is cardiotoxicity due to trastuzumab, which inhibits HER-2 (the pleiotropic molecule) both in the breast tumor cell proliferation context and in the development and protection of heart tissue ${ }^{14}$. Another example is the inhibition of VEGF by tyrosine kinase inhibitors leading to hypertension due to the reduction of the production of NO, among other reasons, with VEGF being the main pleiotropic component in this pathway ${ }^{58}$.

The off-target mechanism is due to the action of the drug in a molecule other than its intended target, but usually bearing a close molecular similarity to it. TKIs are more susceptible to that type of toxicity ${ }^{14}$, one example being the cardiotoxicity due to the inhibition of PDGF (platelet-derived growth factor) by sunitinib, an antiVEGF TKI that impairs cardiomyocyte stress response through the release of pro-angiogenic factors ${ }^{59}$.

It should be pointed out that the aforementioned mechanisms may be simultaneous, for instance, in the case of different types of the same receptor in different tissues. Ultimately, both mechanisms are due to the same fact: even if the target pathway is altered (usually due to hyperexpression), some or all of its components maintain their remaining physiological roles in the body. This highlights the close biological similarity between tumor tissue and normal tissue. Regardless of the mechanism, the toxicity implications are the same as those pointed out relative to traditional chemotherapy drugs, which have been previously described.

\section{CONCLUSIONS}

In this paper we expose a comprehensive view of the processes implicated in chemotherapy treatment, from public health issues concerning diagnostics and drug availability to general molecular pathways involved in drug resistance, especially in multidrug resistance. The distance between such "macroscopic" and "microscopic" scales shows the great complexity of the cancer as group of diseases. All the findings exposed at this text are summarized in the conceptual chart below (Figure 5).

Such distinct issues may rarely be debated in a same context, since their disparities in the scale of investigation. Nonetheless, the integration between those scales is essential to guarantee that cancer patients have access to the best health care, regarding survival and QL, the prime objective of health professionals in the oncology field. Hardly a single person - either a physician, a public health administrator or a scientific investigator - will have influence over more than a few of those factors, and in this lies the need of translations between different fields and expertise towards this common objective. As a broad and accessible reading, we hope that this work serve as and common ground to health professionals and students find new perspectives of investigations.

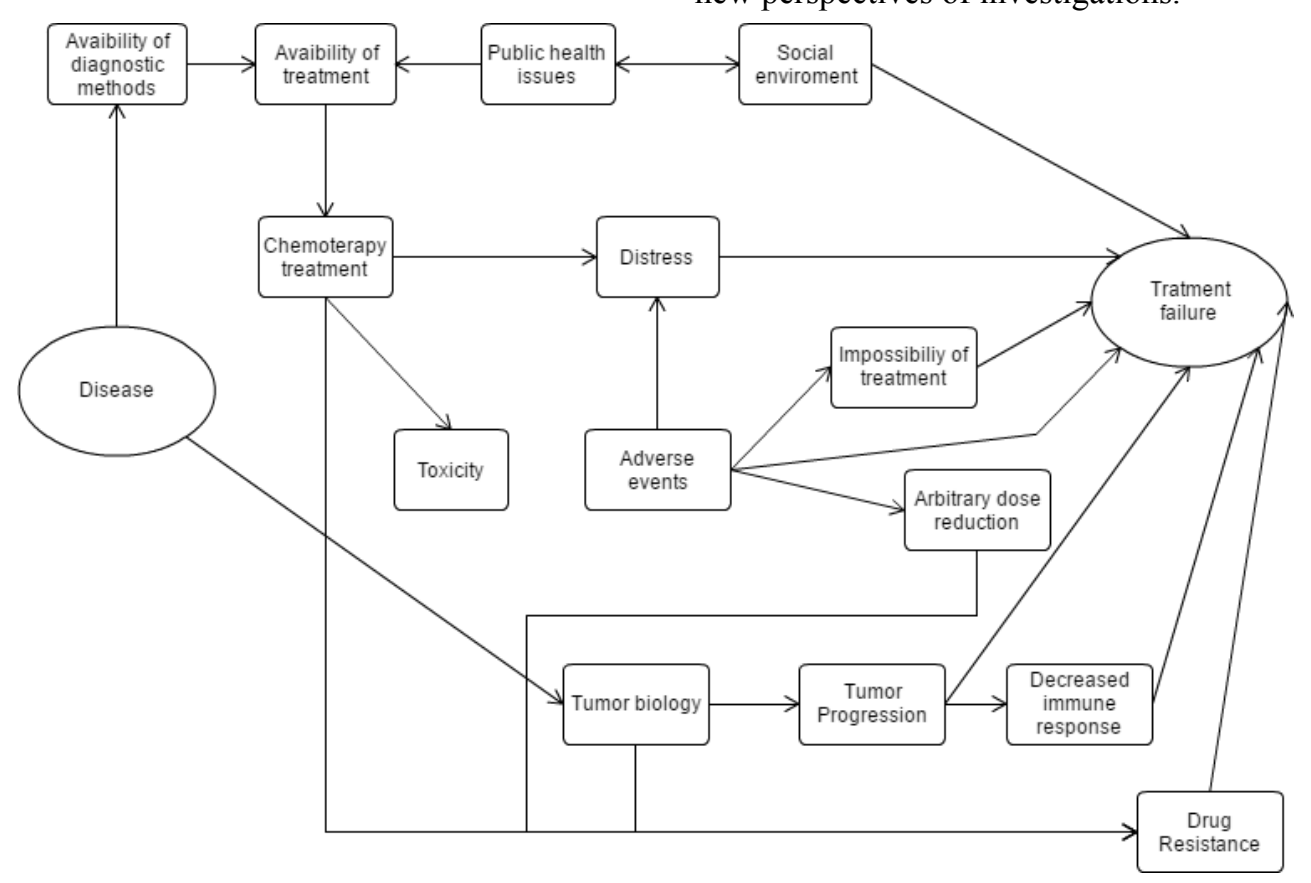

Figure 5. Concepts map illustrating the net of relations between several different concepts exposed previously, that may converge, ultimately, in treatment failure 
Junqueira MZ, Chammas, R. Cancer chemotherapy failure: a synthetic view.

ACKNOWLEDGMENT: The authors thank the academic Carlos Henrique Peres, medicine undergrad student from Faculdade de Medicina da Universidade de São Paulo, due his participation in the early stages of the research that led to the present work.

\section{REFERENCES}

1. American Cancer Society. Cancer Statistic Center. 2017. Available from: https://cancerstatisticscenter.cancer.org/\#!/.

2. Brasil. Ministério da Saúde. Instituto Nacional de Câncer José Alencar Gomes da Silva. Estimativa 2016: incidência de câncer no Brasil. Rio de Janeiro:INCA; 2015. Disponível em: http://www.inca.gov.br/bvscontrolecancer/publicacoes/ edicao/Estimativa_2016.pdf.

3. World Health Organization. Global Health Observatory. 2015. Available from: http://www.who.int/gho/en/.

4. Goldman L, Schafer AI. Goldman-Cecil medicina. Chapter 182, [Abordagem ao paciente com câncer] p.1336-50. 24th ed. Rio de Janeiro: Elsevier; 2014.

5. DeVita VT, Hellman S, Rosenberg SA. Cancer, principles $\&$ practice of oncology. 8th ed. Philadelphia, PA: Lippincott Williams \& Wilkins; 2008. Chap. 22, Sect.1, p.337-43: Principles of medical oncology.

6. Lopes AHI, Castro RM. Oncologia para graduação. 2a ed. São Paulo: Tecmedd; 2008. Cap. 14, p.177-86: Princípios do tratamento sistêmico.

7. Hoff PMG. Tratado de oncologia. São Paulo: Atheneu; 2013. Cap. 45, p.617-30: Princípios de oncologia.

8. Longo DL. Harrison's hematology and oncology. 3rd ed. New York: McGraw-Hill Education Medical; 2017. Chap.27, p.360-73: Approach to the patient with cancer.

9. Sawyers CL. The cancer biomarker problem. Nature. 2008;452(7187):548-52. doi: 10.1038/nature06913.

10. Adler NE, Page A, Institute of Medicine (U.S.). Committee on Psychosocial Services to Cancer Patients/Families in a Community Setting. Cancer care for the whole patient: meeting psychosocial health needs. Washington, D.C.: National Academies Press; 2008. p.26-41.

11. Mimeault M, Hauke R, Batra SK. Recent advances on the molecular mechanisms involved in the drug resistance of cancer cells and novel targeting therapies. Clin Pharmacol Ther. 2008;83(5):673-91. doi: 10.1038/sj.clpt.6100296.

12. Glazer PM, Hegan DC, Lu Y, Czochor J, Scanlon SE. Hypoxia and DNA repair. Yale J Biol Med. 2013;86(4):44351. Available from: https://www.ncbi.nlm.nih.gov/pmc/ articles/PMC3848098/.

13. Wilson WR, Hay MP. Targeting hypoxia in cancer therapy. Nat Rev Cancer. 2011;11(6):393-410. doi: 10.1038/nrc3064.

14. Sodergren SC, Copson E, White A, Efficace F, Sprangers M, Fitzsimmons D, et al. Systematic review of the side effects associated with anti-HER2-targeted therapies used in the treatment of breast cancer, on behalf of the EORTC Quality of Life Group. Target Oncol. 2016;11(3):277-92. doi: 10.1007/s11523-015-0409-2.

15. National Cancer Institute (U.S.). Common terminology criteria for adverse events (CTCAE). Rev. ed. Bethesda, Md.: U.S. Dept. of Health and Human Services, National Institutes of Health, National Cancer Institute; 2009.

14. Sodergren SC, Copson E, White A, Efficace F, Sprangers M, Fitzsimmons D, et al. Systematic Review of the Side Effects
Associated With Anti-HER2-Targeted Therapies Used in the Treatment of Breast Cancer, on Behalf of the EORTC Quality of Life Group. Target Oncol. 2016;11(3):277-92. doi: 10.1007/s11523-015-0409-2.

16. Kerr DJ. Oxford textbook of oncology. 3rd ed. Oxford: Oxford University Press; 2016. Chap. 21, p.186-95: Principles of oncology; p. 186-95.

17. Wood WC, Budman DR, Korzun AH, Cooper MR, Younger $\mathrm{J}$, Hart RD, et al. Dose and dose intensity of adjuvant chemotherapy for stage II, node-positive breast carcinoma. N Engl J Med. 1994;330(18):1253-9. doi: 10.1056/ NEJM199405053301801.

18. Hryniuk WM. Average relative dose intensity and the impact on design of clinical trials. Semin Oncol. 1987;14(1):65-74.

19. Hryniuk WM, Goodyear M. The calculation of received dose intensity. J Clin Oncol. 1990;8(12):1935-7. doi: 10.1200/JCO.1990.8.12.1935.

20. Goldie JH, Coldman AJ. A mathematic model for relating the drug sensitivity of tumors to their spontaneous mutation rate. Cancer Treat Rep. 1979; 63(11-12):1727-33.

21. Norton L, Day R. Potential innovations in scheduling of cancer chemotherapy. Important Adv Oncol. 1991:57-72.

22. Norton L. A Gompertzian model of human breast cancer growth. Cancer Res. 1988;48(24 Pt 1):7067-71.

23. Ignatiadis M, Mavroudis D, Veslemes M, Boukovinas J, Syrigos K, Agelidou M, et al. Sequential versus alternating administration of cisplatin/etoposide and topotecan as first-line treatment in extensive-stage small-cell lung cancer: preliminary results of a Phase III Trial of the Hellenic Oncology Research Group. Clin Lung Cancer. 2005;7(3):183-9. doi: 10.3816/CLC.2005.n.034.

24. DeVita VT, Canellos GP. Combination chemotherapy of solid tumors: an American-Italian collaboration: a celebration of the work of Gianni Bonadonna. Tumori. 2016;102(2):124-6. doi: 10.5301/tj.5000492.

25. Dy GK, Adjei AA. Systemic cancer therapy: evolution over the last 60 years. Cancer. 2008;113(7 Suppl):1857-87. doi: 10.1002/cncr.23651.

26. Goldie JH, Coldman AJ. Application of theoretical models to chemotherapy protocol design. Cancer Treat Rep. 1986;70(1):127-31.

27. Gnoni A, Silvestris N, Licchetta A, Santini D, Scartozzi M, Ria R, et al. Metronomic chemotherapy from rationale to clinical studies: a dream or reality? Crit Rev Oncol Hematol. 2015;95(1):46-61. doi: 10.1016/j.critrevonc.2015.01.008.

28. Kareva I, Waxman DJ, Lakka Klement G. Metronomic chemotherapy: an attractive alternative to maximum tolerated dose therapy that can activate anti-tumor immunity and minimize therapeutic resistance. Cancer Lett. 2015;358(2):100-6. doi: 10.1016/j.canlet.2014.12.039.

29. Goldie JH, Coldman AJ, Gudauskas GA. Rationale for the use of alternating non-cross-resistant chemotherapy. Cancer Treat Rep. 1982;66(3):439-49.

30. Sheiner LB, Rosenberg B, Melmon KL. Modelling of individual pharmacokinetics for computer-aided drug dosage. Comput Biomed Res. 1972;5(5):411-59. 
31. Silva AS, Silva MD, Sudalagunta P, Distler AI, Jacobson T, Collins A, et al. An ex vivo platform for the prediction of clinical response in multiple myeloma. Cancer Res. 2017. doi: 10.1158/0008-5472.CAN-17-0502.

32. Ryan H, Schofield P, Cockburn J, Butow P, Tattersall M, Turner J, et al. How to recognize and manage psychological distress in cancer patients. Eur J Cancer Care (Engl). 2005;14(1):7-15. doi: 10.1111/j.1365-2354.2005.00482.x.

33. Goerling U, Foerg A, Sander S, Schramm N, Schlag PM. The impact of short-term psycho-oncological interventions on the psychological outcome of cancer patients of a surgical-oncology department - a randomized controlled study. Eur J Cancer. 2011;47(13):2009-14. doi: 10.1016/j. ejca.2011.04.031.

34. Mukherjee $\mathrm{S}$. The emperor of all maladies: a biography of cancer. New York: Simon \& Schuster; 2011.

35. Pinquart M, Duberstein PR. Depression and cancer mortality: a meta-analysis. Psychol Med. 2010;40(11):1797810. doi: $10.1017 / \mathrm{S} 0033291709992285$.

36. Sanson-Fisher R, Girgis A, Boyes A, Bonevski B, Burton L, Cook P. The unmet supportive care needs of patients with cancer. Supportive Care Review Group. Cancer. 2000;88(1):226-37.

37. Shabason JE, Mao JJ, Frankel ES, Vapiwala N. Shared decision-making and patient control in radiation oncology: implications for patient satisfaction. Cancer. 2014;120(12):1863-70. doi: 10.1002/cncr.28665.

38. Gupta A, Harris S, Naina HV. The impact of physician posture during oncology patient encounters. J Cancer Educ. 2015;30(2):395-7. doi: 10.1007/s13187-015-0807-2.

39. Andersen BL, Farrar WB, Golden-Kreutz D, Emery $\mathrm{CF}$, Glaser R, Crespin T, et al. Distress reduction from a psychological intervention contributes to improved health for cancer patients. Brain Behav Immun. 2007;21(7):95361. doi: 10.1016/j.bbi.2007.03.005.

40. McGregor BA, Antoni MH. Psychological intervention and health outcomes among women treated for breast cancer: a review of stress pathways and biological mediators. Brain Behav Immun. 2009;23(2):159-66. doi: 10.1016/j. bbi.2008.08.002.

41. Thaker PH, Han LY, Kamat AA, Arevalo JM, Takahashi $\mathrm{R}, \mathrm{Lu} \mathrm{C}$, et al. Chronic stress promotes tumor growth and angiogenesis in a mouse model of ovarian carcinoma. Nat Med. 2006;12(8):939-44. doi: 10.1038/nm1447.

42. Williams JB, Pang D, Delgado B, Kocherginsky M, Tretiakova M, Krausz T, et al. A model of gene-environment interaction reveals altered mammary gland gene expression and increased tumor growth following social isolation. Cancer Prev Res (Phila). 2009;2(10):850-61. doi: 10.1158/1940-6207.CAPR-08-0238.

43. Dietze EC, Sistrunk C, Miranda-Carboni G, O'Regan R, Seewaldt VL. Triple-negative breast cancer in AfricanAmerican women: disparities versus biology. Nat Rev Cancer. 2015;15(4):248-54. doi: 10.1038/nrc3896.

44. Levy EM, Roberti MP, Mordoh J. Natural killer cells in human cancer: from biological functions to clinical applications. J Biomed Biotechnol. 2011;2011:676198. doi: $10.1155 / 2011 / 676198$

Recebido em:19.08.17

Aceito em: 13.03.18
45. Schantz SP, Goepfert H. Multimodality therapy and distant metastases. The impact of natural killer cell activity. Arch Otolaryngol Head Neck Surg. 1987;113(11):1207-13.

46. Hulbert-Williams NJ, Storey L. Psychological flexibility correlates with patient-reported outcomes independent of clinical or sociodemographic characteristics. Support Care Cancer. 2016;24(6):2513-21. doi: 10.1007/s00520-0153050-9.

47. Kelloff GJ, Sigman CC. Cancer biomarkers: selecting the right drug for the right patient. Nat Rev Drug Discov. 2012;11(3):201-14. doi: 10.1038/nrd3651.

48. Ludwig JA, Weinstein JN. Biomarkers in cancer staging, prognosis and treatment selection. Nat Rev Cancer. 2005;5(11):845-56. doi: 10.1038/nrc1739.

49. Vieira S. Bioestatística - tópicos avançados. 3a ed. Rio de Janeiro: Elsevier; 2010. Cap. 8, Sec.8.2 Exames diagnósticos.

50. Ferreira CG, Achatz MI, Ashton-Prolla P, Begnami MD, Marchini FK, Stefani SD. Brazilian health-care policy for targeted oncology therapies and companion diagnostic testing. Lancet Oncol. 2016;17(8):e363-70. doi: 10.1016/ S1470-2045(16)30171-1.

51. Rehman A, Awais M, Baloch NU. Precision medicine and low- to middle-income countries. JAMA Oncol. 2016;2(3):293-4. doi: 10.1001/jamaoncol.2015.5511.

52. Retsky MW, Swartzendruber DE, Wardwell RH, Bame PD. Is Gompertzian or exponential kinetics a valid description of individual human cancer growth? Med Hypotheses. 1990;33(2):95-106.

53. Benzekry S, Lamont C, Beheshti A, Tracz A, Ebos JM, Hlatky L, et al. Classical mathematical models for description and prediction of experimental tumor growth. PLoS Comput Biol. 2014;10(8):e1003800. doi: 10.1371/ journal.pcbi.1003800.

54. Rang HPR, Flower RJ, Henderson G. Rang \& Dale Pharmacology. 8th ed. New York: Elsevier; 2016. Chap. 56, p.676-90: Antineoplasic agents.

55. Albini A, Sporn MB. The tumour microenvironment as a target for chemoprevention. Nat Rev Cancer. 2007;7(2):13947. doi: $10.1038 / \mathrm{nrc} 2067$.

56. Dbaibo GS, Pushkareva MY, Rachid RA, Alter N, Smyth MJ, Obeid LM, et al. p53-dependent ceramide response to genotoxic stress. J Clin Invest. 1998;102(2):329-39. doi: 10.1172/JCI1180.

57. Weinberg RA. The biology of cancer. 2nd ed. New York: Garland Science, Taylor \& Francis Group; 2013. Chap. 2, p.31-70: The nature of cancer.

58. Curigliano G, Cardinale D, Dent S, Criscitiello C, Aseyev $\mathrm{O}$, Lenihan D, et al. Cardiotoxicity of anticancer treatments: Epidemiology, detection, and management. CA Cancer $\mathrm{J}$ Clin. 2016;66(4):309-25. doi: 10.3322/caac.21341.

59. Maurea N, Coppola C, Piscopo G, Galletta F, Riccio G, Esposito E, et al. Pathophysiology of cardiotoxicity from target therapy and angiogenesis inhibitors. J Cardiovasc Med (Hagerstown). 2016;17 Suppl 1:S19-26. doi: 10.2459/ JCM.0000000000000377. 